\title{
Propiedad, democracia y monarquía en John Locke. (¿Era Locke un partidario de la igualdad política y la democracia?)
}

\author{
Property, Democracy \\ and Monarchy in John Locke. \\ Was Locke a Supporter \\ of Political Equality and Democracy? \\ ROBERTO RODRÍGUEZ GUERRA \\ Universidad de La Laguna
}

Recibido: 07-VII-2014 Aprobado definitivamente: 20-XI-2014

\section{RESUMEN}

El presente trabajo discute aquellas interpretaciones de Locke como un continuador del radicalismo leveller y un partidario inequívoco de la igualdad política y la democracia. Sostiene que su obra y pensamiento político persigue más bien un retorno a «our ancient government» $\mathrm{y}$ «its original constitution», esto es, un modelo de «monarquía moderada» o «mixta» que no sólo representa un retroceso democrático respecto al «republicanismo popular» de los levellers sino también una forma de gobierno en la que los elementos democráticos e igualitarios quedan debilitados.

\section{PALABRAS CLAVE \\ LOCKE, LIBERALISMO-IGUALDAD-DEMOCRACIA-MONARQUÍA}

\section{ABSTRACT}

This paper questions those interpretations of Locke as a follower of leveller radicalism and an unequivocal supporter of political equality and democracy. It argues that his work and political thought seek mainly a return to «our ancient government» and «its original constitution», that is, 
a model of "moderate or mixed monarchy" which not only represents a democratic regression compared to the «popular republicanism» of the Levellers but also a form of government in which the democratic and egalitarian elements are weakened.

\section{KEY WORDS \\ LOCKE-LIBERALISM-EQUALITY-DEMOCRACY-MONARCHY}

Si LOS LEVELLERS CONSTITUYERON un primer exponente del constitucionalismo moderno y liberal, ${ }^{1}$ Locke fue -como es de sobra reconocido- el gran representante del pensamiento político moderno en cuya obra se inspiran y esbozan los elementos ideopolíticos básicos de la tradición política liberal. ${ }^{2} \mathrm{De}$ él se ha dicho que fue el «alma», ${ }^{3}$ «padre» ${ }^{4} \mathrm{o}$ «fundador» ${ }^{5}$ del liberalismo, así como que fue «el teórico» ${ }^{6}$ o «principal ideólogo de la revolución inglesa de $1688 »{ }^{7}$ Pero también se ha dicho -y esto es lo que aquí nos interesa- que fue un partidario inequívoco de la igualdad política y la democracia. ${ }^{8}$ Ciertamente, tal afirmación parece formar parte de aquella línea interpretativa del pensamiento de Locke que-ya antes de mediados del pasado siglo XX- lo considera «un demócrata partidario del dominio de la mayoría», ${ }^{9}$ al tiempo que nos ofrece una imagen del mismo como un intelectual que continúa el radicalismo leveller y fundamenta la vindicación de la soberanía popular, la extensión del sufragio

1 D. Wootton, «The Levellers», en J. Dunn, ed., Democracy: the unfinished journey, 508 $B C$ to AD 1993. Oxford: Oxford University Press, 1992, 71.

2 R.W. Grant, John Locke's Liberalism. Chicago: University of Chicago Press, 1987; Sh. Wolin, Política y perspectiva. Continuidad y cambio en el pensamiento político occidental. BBAA: Amorrortu, 1973, 313.

3 J. Plamenatz, Man \& Society. From the Middle Ages to Locke, London: Longman, 1992, vol. I, 377.

4 H.C. Mansfield, Taming the Prince. The Ambivalence of Modern Executive Power. Baltimore: Johns Hopkins University Press, 1993.

5 J.E. Parsons, «Locke's doctrine of property», Social Research, 36, 1969, 392.

6 J. Plamenatz, Political Thought in England from Locke to Bentham. London: Williams \& Norgate, 1920, 29.

7 J.M. Colomer, «Ilustración y liberalismo en Gran Bretaña: J. Locke, D. Hume, los economistas clásicos, los utilitaristas», en F. Vallespín, ed., Historia de la teoría política, Madrid: Alianza, 1991, vol. 3, 13 y s.

8 R. Dahl, «Democracy», Encyclopaedia Britannica, disponible en http://www.britannica. com/_Bchecked/topic/157129/democracy.

9 Esta era, si bien desde una óptica conservadora, la interpretación de Willmoore Kendall en su John Locke and the Doctrine of Mayority-Rule. Illinois: University of Illinois Press, 1941, 103 y ss. 
$\mathrm{y}$, en suma, la defensa de una forma de gobierno democrático-representativa. ${ }^{10}$ Pero tal interpretación de la teoría política de Locke ${ }^{11}$ tropieza con más de un problema, en especial porque en nuestra opinión su obra y pensamiento político pretende -como él mismo sugirió ${ }^{12}$ - un retorno a «nuestro antiguo gobierno» y su «constitución original». No obstante, desvelar el concreto significado de dicho «retorno» y relacionarlo problemáticamente con la imagen de Locke antes citada exige acercarnos - si bien con la brevedad exigida- a las bases de su pensamiento político, esto es, a sus teorías de la propiedad, de la sociedad política, de las formas de gobierno y, en definitiva, a su concreta concepción de la democracia y la monarquía.

\section{PROPIEDAD, CIUDADANÍA Y SOCIEDAD POLÍTICA EN LOCKE}

Como es sabido, la teoría política de Locke -a la que Macpherson ha vinculado al marco ideológico del individualismo posesivo ${ }^{13}$ - parte del principio

10 Cf. R. Ashcraft, Revolutionary Politics and Locke's Two Treatises of Government. Princeton: Princeton University Press, 1986, 78; del mismo Locke's Two Treatises on Government. London: Allen \& Unwin, 1987; J.H. Franklin, John Locke and the Theory of Sovereignty: Mixed Monarchy \& the Right of Resistance in the Political Thought of the English Revolution. Cambridge: Cambridge University Press, 1978. Un acercamiento crítico a esta imagen de Locke puede encontrarse en D. McNally, «Locke, Levellers and Liberty: Property and Democracy in the Thoughts of the First Whigs», History of Political Thought, X, I, 1989, 17-40. Véase también E. Meiksins Wood, «Locke against Democracy: Consent, Representation and Suffrage in the Two Treatise» (History of Political Thought, XIII, 4, 1992, 657-689) y «Radicalism, Capitalism and Historical Contexts. Not only a Replay to Richard Ashcraft» (History of Political Thought, XV, 3, 1994, 323-372).

11 Un primer acercamiento a las diversas interpretaciones de la obra y pensamiento de Locke puede encontrarse en R. Ashcraft, R., «A Critical Note on Locke Scholarship» (op. cit., 1987, 298-305).

12 En carta dirigida a Edward Clarke en febrero de 1689 («Letter to Edward Clarke», en Political Writings. London: Penguin Books, 1993, 437) Locke, ante el intento de reordenar el sistema político inglés tras la huida de Jacobo II, sostiene que se estaba ante la magnífica oportunidad de «encontrar remedios y establecer una constitución que puede ser perdurable para la seguridad de los derechos civiles, la libertad y la propiedad». Y seguidamente afirma que el mejor modo de hacerlo es «restaurando nuestro antiguo gobierno, posiblemente el mejor que alguna vez hubo, si se toma y se reorganiza todo en una sola pieza en su constitución original».

13 C.B. Macpherson, La teoría politica del individualismo posesivo: Barcelona: Fontamara, 1979, 169 y ss. Un acercamiento crítico al enfoque de Macpherson puede verse, entre otros, J. Dunn, J. The Political Thought of John Locke. Cambridge, Cambridge University Press: 1968, 214 y ss.; D. Miller, «The Macpherson versión», Political Studies, XXX, 1, 1982, 120-127; o J. Tully, An Approach to Political Philosophy: Locke in Contexts. Cambridge: Cambridge University Press, 1993, 71 y ss. Por lo demás, una respuesta a la crítica de Miller, Tully y otros a Macpherson puede encontrarse en J. Townshend, C. B. Macpherson and the Problem of Liberal 
de que el hombre «tiene por naturaleza el poder de proteger su propiedad, es decir, su vida, libertad y sus bienes». Un principio que complementa con aquel otro según el cual al ser los hombres «libres por naturaleza, iguales e independientes, ninguno puede ser sacado de esa condición y puesto bajo el poder político de otro sin su propio consentimiento»». ${ }^{14}$ Lo que origina y de hecho constituye una sociedad política no es pues, para Locke, otra cosa que «el consentimiento de una pluralidad de hombres libres que aceptan la regla de la mayoría», dando origen con ello a la articulación de «un cuerpo político bajo un gobierno supremo»». ${ }^{15}$ Resulta pues de especial interés para nuestros propósitos determinar quién constituye en realidad esa «pluralidad de hombres libres» que ha de dar su consentimiento para la constitución de la sociedad política. Pero esa será una cuestión que habremos de dilucidar poco más adelante. Por el momento ha de señalarse que en su pensamiento el paso del estado de naturaleza a la sociedad civil o política tiene por finalidad evitar los inconvenientes que el primero implica para los individuos y, en último término, «preservar sus vidas, sus libertades y sus posesiones, es decir, todo eso a lo que doy el nombre genérico de "propiedad"»». ${ }^{16}$

La protección de la «propiedad» se constituye pues en Locke como fin de la sociedad política y, al mismo tiempo, como límite del alcance y funciones del poder político. Pero también como instrumento clave para la regulación de las relaciones propias de una sociedad basada en el capitalismo agrario ${ }^{17}$-con la cual Locke estaba claramente familiarizado en tanto propietario ${ }^{18} \mathrm{y}$ miembro de diversas instituciones relacionadas con el comercio ${ }^{19}$ - y en unas relaciones socio-económicas crecientemente mercantiles a las que se pretendía liberar de la intervención «no protectora» del Estado. El mismo Locke lo indica con

\section{Democracy. Edinburgh: Edinburgh University Press, 2000.}

14 J. Locke, Segundo Tratado sobre el Gobierno Civil. Madrid: Técnos, 2006, 87 y 95 (traducción, introducción y notas de Carlos Mellizo y «Estudio Preliminar» de Peter Laslett).

15 Ibid., 99.

16 Ibid., 123. En su Carta sobre la tolerancia de 1685 (en Ensayo y Carta sobre la tolerancia. Madrid: Alianza, 1999, 66) Locke sostiene acaso con mayor precisión que «el Estado es, a mi parecer, una sociedad de hombres constituida únicamente para preservar y promocionar sus bienes civiles. Lo que llamo bienes civiles son la vida, la libertad, la salud corporal, el estar libre de dolor y la posesión de cosas externas tales como dinero, tierras, casas, muebles y otras semejantes»

17 Cf. Neal Wood, John Locke and Agrarian Capitalism. Berkeley: University of California Press, 1984.

18 Para la considerable extensión de los bienes de Locke puede consultarse M. Cranston, Locke: A Biography. Oxford: Oxford University Press, 1985, 114 y s., 377, 448 y 475.

19 Cf. M. Cranston (op. cit., 153 y ss.), J. Tully (op. cit., 63) o David Armitage («John Locke, Carolina, and the 'Two Treatises of Government'», Political Theory, 32, 2004, 603 y ss.). 
toda claridad cuando afirma que la protección de la «propiedad» no es sólo el medio para «limitar el poder» del Estado. Es también una forma de «moderar el dominio que cada miembro o parte de esa sociedad pueda tener sobre los demás». ${ }^{20}$ Con ello Locke tiende a reflejar las aspiraciones y mentalidad de una pujante sensibilidad y clase social a la que estaba claramente vinculado y que-visiblemente representada por los Whigs- aspira a la construcción de una nueva sociedad y poder político fundada sobre principios e instituciones que posteriormente se conceptualizaron como «liberales».

Es sabido que a tales efectos Locke parte de una justificación del derecho «natural»a la apropiación privada de las cosas a partir del trabajo para, en un segundo momento e indirectamente, eliminar los límites a tal derecho de apropiación y justificar por tal vía un derecho ilimitado a la apropiación privada. ${ }^{21}$ En todo caso, «la asombrosa hazaña de Locke consistió en fundamentar el derecho de propiedad en el derecho natural y en la ley natural, y en eliminar luego todos los límites del derecho natural para la propiedad privada». ${ }^{22}$ No obstante, para nuestros propósitos interesa destacar aquí que la concepción lockeana del derecho de propiedad posee al menos dos importantes matices adicionales. El primero de ellos reside en que Locke concibe el derecho de propiedad como un título estrictamente individual y exclusivo sobre las cosas, esto es, como un derecho que otorga un «exclusivo dominio privado» ${ }^{23}$ sobre las posesiones de cada cual. Tal título, por una parte, confiere al apropiador el derecho a excluir a otros del uso y/o disfrute de aquello que se ha apropiado y, por otra, exime al poseedor de casi toda obligación social que pudiera derivarse del mismo acto de apropiación. Y todo ello sin que la apropiación privada de las cosas que son originariamente comunes dependa de «un acuerdo expreso entre los miembros de la comunidad». ${ }^{24}$ De esta forma, y tras la ya señalada eliminación de los límites a la apropiación privada de las cosas comunes y especialmente por medio del artificio del dinero, el derecho ilimitado a la propiedad privada se transforma no sólo en una nueva forma de propiedad privada. También se constituye en una forma de relación social que, por un lado, avala la acumulación de posesiones o riquezas ${ }^{25}$ al tiempo que justifica las profundas

20 J. Locke, Segundo Tratado, 222.

21 Cf. la prolija y detallada argumentación de Macpherson (op. cit., 169-223).

22 Ibid. 173.

23 J. Locke, Segundo Tratado, 26.

24 Ibid., 25. La teoría de la propiedad de Locke (Macpherson, op. cit., 190 y s.) no sólo da una base moral a la apropiación burguesa y a la sociedad capitalista. También minó aquella «concepción tradicional según la cual la propiedad y el trabajo eran funciones sociales, y la propiedad implicaba obligaciones sociales».

25 «Tan pronto como un hombre descubre que hay algo que tiene el uso y el valor del dinero en sus relaciones con sus vecinos, veremos que ese hombre empieza a aumentar sus 
desigualdades sociales generadas por tal proceso de acumulación y, por otro, permite -y en realidad- exige la libertad e iniciativa individual, la separación entre economía y política, la supremacía de la economía y las relaciones de mercado sobre la política y el Estado, la intervención fundamentalmente «protectora» de éste sobre aquella y la consiguiente limitación del alcance y los fines del gobierno. Se constituye, en síntesis, en el fundamento real de las relaciones sociales capitalistas y en un severo límite a los fines y el alcance del poder y las instituciones políticas. ${ }^{26}$

Por su parte, el segundo de los matices a que más arriba aludíamos reside en que -como argumentan Macpherson o Plamenatz ${ }^{27}$ - Locke maneja de hecho dos sentidos diferentes del concepto de propiedad. El primero es el ya señalado concepto genérico que incluye «la vida, libertad y posesiones individuales». El segundo es un concepto restringido según el cual la idea de propiedad alude específicamente a los bienes y la tierra, esto es, a «la posesión de cosas externas tales como dinero, tierras, casas, muebles y otras semejantes». Y lo decisivo a este respecto es que-como ya se sugería poco antes - mientras Locke utiliza el concepto «genérico» de propiedad para dar cuenta del paso del estado de naturaleza a la sociedad civil o política, recurre sin embargo al concepto «restringido» de propiedad para definir quiénes pueden ser ciudadanos de pleno derecho en la sociedad política ya constituida. Todos los miembros de la comunidad -insiste Locke- deben dar su consentimiento al pacto que consolida el paso del estado de naturaleza a la constitución de la sociedad política. Veremos más adelante las formas en que para Locke puede expresarse tal consentimiento. Por ahora conviene destacar que para Locke, una vez constituida la sociedad política, no todos tienen derecho a elegir a aquellos que han de ejercer el poder político o a ser elegidos para ejercerlo. Los carentes de hacienda y propiedad (mendigos, sirvientes, desempleados, trabajadores) no parecen ser considerados por Locke como ciudadanos o miembros de pleno de derecho de la sociedad política ya constituida. Es esta ambigüedad y doble uso del concepto de propiedad lo que según Macpherson «permite a Locke considerar a todos los hombres como miembros al objeto de ser gobernados y solamente a los hombres con hacienda como miembros al objeto de gobernar. El derecho a gobernar (más exactamente el derecho a controlar al gobierno) sólo se concede a los hombres con hacienda: son ellos quienes tienen el voto decisivo en la cuestión de los impuestos, sin los cuáles ningún gobierno puede subsistir». ${ }^{28}$ Por lo demás, a tales restricciones

posesiones» (J. Locke, Segundo Tratado, 49).

26 Desde esta perspectiva Locke aparece-como entre otros ha señalado M. Cranston (op. cit., 211)- como uno de los primeros defensores del Estado mínimo.

27 C.B. Macpherson (op. cit., 211 y ss.) y J. Plamenatz (op. cit., 1992, 338).

28 C.B. Macpherson, ob. cit., 121 y s. Una más amplia discusión acerca de la extensión del sufragio en Locke, así como de sus relaciones con los levellers, puede encontrarse en Meiksins 
habría que añadir no sólo la consabida exclusión de las mujeres, esclavos e indígenas. También cabe presumir que, dados los límites lockeanos al principio de tolerancia, los ateos, católicos, protestantes milenaristas o los musulmanes podrían estar igualmente excluidos -al menos en cuanto a su expresión público-política se refiere- de tales derechos en tanto que posibles portadores de opiniones y/o acciones públicas que son «contrarias a la sociedad humana o a las reglas morales que son necesarias para la preservación de la sociedad civil», esto es, en tanto defensores de opiniones «destructivas para la sociedad» y perturbadoras de la seguridad del Estado. ${ }^{29}$ Tales son pues las restrictivas bases sobre las que realmente se sustenta la igualdad política y toda forma de gobierno en Locke. Y de ahí que pueda decirse - con J. Plamenatz- que «Locke no fue un creyente en la igualdad, ya sea social o política». ${ }^{30}$

\section{Poder Político, GOBIERNo LIMITADO Y DESPOTISMO}

Lo dicho hasta el momento implica una respuesta negativa a la pregunta de si era Locke un partidario inequívoco de la igualdad, al tiempo que muestra algunos de los rasgos del pensamiento político lockeano. Pero no acaba en cambio de perfilar adecuadamente la cuestión de la concreta forma de gobierno de la que a su juicio ha de dotarse una comunidad política. Aclarar tal cuestión exige empero detenernos en otros aspectos de su teoría política. Y tal efecto ha de tenerse presente, en primer lugar, que para Locke el poder legislativo es el «poder supremo,..., sagrado e inalterable». ${ }^{31}$ Ahora bien, dicho poder ha de gobernar «según normas y reglas establecidas, imparciales y aplicables a todos por igual» o-como dice en otro momento- «según lo que dicten las leyes establecidas, promulgadas y conocidas del pueblo». ${ }^{32}$ Es más, Locke sostiene que tal poder no puede ser ejercido de modo absoluto y arbitrario sobre «las vidas y fortunas del pueblo». Tampoco puede gobernar mediante «decretos arbitrarios y extemporáneos», ni «apoderarse de parte alguna de la propiedad de un hombre sin el consentimiento de éste» (si bien debe «hacer leyes para

Wood (op. cit., 1992, 657-689 y 1994, 323-372).

29 Cf. J. Locke, Ensayo sobre la tolerancia de 1666 (op. cit, 39 y ss. y 46 y ss.) o, a modo de resumen, las restricciones al principio de tolerancia que establece en su Carta sobre la tolerancia de 1685 (op. cit., 108-110). Por lo demás no consta que Locke se opusiera al Test Act de 1673 por el cual se excluía a los católicos y disidentes del desempeño de cargos públicos a menos que apostasen de su fe. Es más, en este mismo sentido abundan sus vínculos con John Ashley Cooper y su apoyo a los sucesivos proyectos de Ley de Exclusión presentados al Parlamento durante la Crisis de Exclusión.

30 J. Plamenatz, op. cit.,1992, 333.

31 J. Locke, Segundo Tratado, 134.

32 Ibid., 87 y 131. 
regular la propiedad entre los súbditos»), ni «transferir a nadie el poder de hacer leyes». ${ }^{33}$ De ahí que el poder legislativo sea en Locke -pese a que lo considera el «poder supremo-un poder estrechamente vinculado con la tutela de los derechos de «propiedad» de los individuos.

En todo caso, de lo anterior es posible colegir que la defensa de una forma de gobierno limitado y conforme a (y mediante) leyes se convierte en otro de los aspectos centrales de la teoría política lockeana. Pero de lo que se acaba de señalar puede deducirse igualmente que para Locke todo «poder despótico»-al que define como «un poder absoluto y arbitrario que un hombre ejerce sobre otro... [y que] la naturaleza jamás concede» ${ }^{34}$ - «está tan lejos de ser compatible con la sociedad civil que se opone a ella en la misma medida en que la esclavitud se opone a la propiedad». ${ }^{35} \mathrm{Y}$ da lo mismo que dicho poder se ejerza bajo la forma de una monarquía absoluta ${ }^{36}$ o de una tiranía. ${ }^{37}$ Del mismo modo que da igual que tal poder tenga su origen en la conquista ${ }^{38}$ o en la usurpación. ${ }^{39}$ Ninguna forma despótica de gobierno es compatible para Locke con los derechos naturales individuales ni con el fundamento que el consentimiento de cada uno da a la constitución de la sociedad política.

\section{DE LA DEMOCRACIA PERFECTA AL GOBIERNO REPRESENTATIVO}

Excluidas tales posibilidades Locke cree sin embargo que las formas de gobierno de que finalmente puede dotarse una comunidad pueden ser varias y dependen, en definitiva, «de dónde se deposite el poder supremo, que es el legislativo». Indica así mismo que «sólo el pueblo puede determinar el tipo de Estado, lo cual hace constituyendo el poder legislativo e indicando en qué manos ha de estar». ${ }^{40}$ Es más, precisa en tal sentido que la mayoría puede emplear todo su poder a fin de «hacer periódicamente leyes para la comunidad,

33 Ibid., 135-142.

34 Ibid., 172.

35 Ibid., 174.

36 Que para Locke (Ibid., 91 y 94) presupone tanto la existencia de alguien -el príncipeque «está exento de las reglas y normas de la sociedad civil» como que «no hay nadie a quien [los hombres] puedan apelar frente a los daños que tal individuo les puede causar».

37 La cual (Ibid., 199) «es un poder que viola lo que es de derecho» y que se ejerce «no para el bien de quienes están bajo ese poder, sino para propia ventaja de quien lo ostenta».

38 Que implica (Ibid., 175) un gobierno establecido «por la fuerza de las armas,..., pero sin el consentimiento del pueblo».

39 Que es (Ibid., 197) «una suerte de conquista doméstica» por la cual «uno ha tomado posesión de algo a lo que no tiene derecho».

40 Ibid.141. 
y en ejecutar esas leyes sirviéndose de los oficiales que la mayoría nombra». De ser así estaríamos ante una «democracia perfecta». ${ }^{41}$

Por lo que aquí nos interesa cabe señalar que Locke no aporta mayores consideraciones respecto de la «democracia perfecta». No obstante, de lo ya indicado y de otros aspectos de su pensamiento político puede inferirse que rechaza abiertamente tal forma de gobierno. En efecto, cabe pensar en primer lugar que los mismos fines y límites que Locke establece para toda forma de poder político son igualmente aplicables a la «democracia perfecta». Y esto, en la concepción lockeana, excluye la admisibilidad de una forma de gobierno en la que la mayoría puede usar su poder de modo irrestricto o ilimitado o, cuando menos, de modo que suponga algún peligro para la «propiedad» de los individuos. Por otra parte, es sabido que Locke -pese a que sostiene que nadie puede ser colocado bajo un poder político sin su consentimiento- no cree necesario que cada cual -y en conjunción con los demás- haya de dar su expreso y efectivo consentimiento para la constitución de la sociedad política. A su juicio basta a este respecto con el «consentimiento tácito» que todo hombre expresa desde el momento en que «tiene posesiones o disfruta de alguna parte de los dominios de un gobierno» o, en suma, por «el mero hecho de estar dentro de los territorios de ese gobierno». ${ }^{42}$ Es más, en Locke el consenso por el cual se establece un gobierno, de un lado, es «casi imposible» que sea absoluto y tan solo debe ser «de la mayoría de esa sociedad» y, de otro, «lo da cada individuo por separado (cuando le llega el turno de su mayoría de edad), y no lo dan todos juntos a una misma vez». ${ }^{43}$ De ahí que pueda decirse, por una parte, que en Locke queda excluido -incluso en el momento «constituyente»- el ejercicio directo de la participación política ciudadana y, por otra, que en su perspectiva, al igual que en la de muchos otros de sus coetáneos, la «democracia perfecta» es tan solo una mera posibilidad teórica y no una forma de gobierno deseable para su época y sociedad. ${ }^{44}$

41 Ibid., 132.

42 Ibid., 117.

43 Ibid, 96-98 y 117.

44 Del rechazo de Locke a la democracia es igualmente sintomático el diseño de la estructura política que, en colaboración con Shaftesbury, propone en The Fundamental Constitutions of Carolina (Political Writings, 210-231). Una propuesta que, por un lado, era propia de una mentalidad colonial que perseguía la expansión y la protección de los intereses de los grandes terratenientes a quienes la corona inglesa había cedido la provincia de Carolina; por otro, asumía la existencia de la esclavitud, otorgaba a los poseedores de esclavos el poder absoluto sobre la vida y la muerte de los mismos y establecía la primera nobleza hereditaria en suelo norteamericano; y, por último, estaba expresamente destinada a «evitar una democracia numerosa» (211). Para una aproximación al «giro colonial» en los recientes estudios sobre el pensamiento de Locke véase, entre otros, J. Tully (op. cit., 137 y ss.) y David Armitage (op. cit., 602-627). 
Descartadas pues tanto la «democracia perfecta» como cualquier forma de poder político despótico queda aún por esclarecer cuál era la forma de gobierno preferida por Locke. Ciertamente, no es esta una tarea fácil pues -como entre otros ha señalado J. Plamenatz ${ }^{45}$ - el pensamiento político de Locke y, especialmente, su Segundo Tratado, no constituye una clara apología de una particular forma de gobierno. Pero, pese a ello, nos gustaría sugerir aquí que las consideraciones lockeanas a este respecto apuntan hacia la justificación de alguna forma de «gobierno representativo» $\mathrm{y}$ «moderado». Ahora bien, como es sabido, para Locke este tipo de gobierno podría articularse de diversos modos $\mathrm{y}$, particularmente, mediante cierta combinación de las tres formas «puras» de gobierno. De hecho, es a una peculiar suerte de «gobierno mixto» a la que Locke -como en adelante veremos- ofrece su apoyo y al que concibe al modo de una «monarquía moderada».

\section{Del Gobierno RePRESENTATIVo A LA MONARQuía MODERADA}

En opinión de Locke toda forma adecuada de gobierno-al igual que ocurre en los «gobiernos bien estructurados»- debe, entre otras cuestiones, preservar cierta forma de separación de poderes. Ahora bien, conviene adelantar aquí, por una parte, que será su peculiar concepción de la separación de poderes la que nos ofrezca más de un indicio acerca de cuál es la forma adecuada de gobierno por la que apuesta y, por otra, que si bien distingue entre "poder legislativo», «poder ejecutivo»y «poder federativo» -además del «poder de prerrogativa»tan solo aplica el principio de separación de poderes $-\mathrm{y}$, aun así, con ciertos matices- a los poderes ejecutivo y legislativo, de los que dice que deben estar «en distintas manos». ${ }^{46}$

Según Locke, el poder legislativo podría estar en manos de una asamblea «compuesta de representantes que son escogidos por el pueblo». Dicha representación habría de ser justa y equitativa, «proporcional a la población a la que dichos representantes sirven $» .{ }^{47}$ En todo caso, tal asamblea «tiene el poder de hacer leyes» y, realizada tal tarea, «vuelve a disolverse», puesto que las leyes necesarias «pueden ser hechas en muy poco tiempo». ${ }^{48} \mathrm{El}$ poder legislativo se ejerce así para Locke «a intervalos» o cuando se crea necesario reunirlo, pero no de modo permanente, pues esto podría conducirlo a extender su alcance y fines o, en suma, a ocuparse de las tareas de gobierno y, de este modo, asumir funciones del poder ejecutivo.

45 J. Plamenatz, op. cit., 1992, 331.

46 J. Locke, Segundo Tratado, 159.

47 Ibid, 158.

$48 \mathrm{Ibid}, 143$. 
Por su parte, el poder ejecutivo debe estar siempre subordinado al legislativo y, en todo caso, al poder del pueblo (el cual siempre retiene «el supremo poder de disolver o de alterar la legislatura»). ${ }^{49} \mathrm{Al}$ mismo tiempo, según Locke, es preciso «que esté siempre en activo y vigile la puesta en práctica de las leyes y la aplicación de las mismas». ${ }^{50}$ Pero -lo que es más relevante a nuestro efectos- nuestro autor afirma igualmente que el poder ejecutivo también debería ostentar el poder de «prerrogativa», esto es, el poder discrecional de legislar -para bien de la comunidad y hasta tanto el legislativo no lo haga- sobre aquellas cuestiones no previstas por la ley y, lo que es más, «sin hacerlo conforme a lo prescrito por la ley, aún contra ella en ciertos casos». ${ }^{51} \mathrm{El}$ poder ejecutivo contemplado por Locke implica así un poder que está autorizado, si bien bajo ciertas restricciones, a legislar. Pero, del mismo modo, para Locke es igualmente recomendable que el ejecutivo ostente el «poder federativo», esto es, la dirección de las relaciones con otros Estados, la capacidad «de hacer la guerra y la paz, de establecer ligas y alianzas y de realizar tratos con todas las personas y comunidades fuera del Estado». ${ }^{52}$

Pero ¿cómo concreta Locke el modelo constitucional que parece derivarse de tales premisas? Las anteriores indicaciones parecen dirigirse hacia la defensa de alguna forma de gobierno representativo «puro», si bien -a tenor de los límites a los derechos políticos más arriba señalados- tal tipo de gobierno poseería un carácter propietarista y restrictivo. No obstante, no son pocas las sugerencias de Locke que tiende a debilitar tal posibilidad. Es cierto que en su obra existe cierta ambigüedad teórica al respecto. Pero, en nuestra opinión, de lo ya señalado-además otras cuestiones a las que seguidamente aludiremos- se deriva un claro intento de justificar una concreta forma de «gobierno mixto» $\mathrm{y}$, más concretamente aún, de «monarquía moderada». De hecho sostiene en tal sentido no solo que «no puede haber constitución más sabia» que aquella en la que «por ley, la persona del príncipe es sagrada... y está libre de toda cuestión o violencia». ${ }^{53}$ También propone una forma de gobierno en la que el príncipe o

49 Ibid, 149 y 168. Estamos pues ante la teoría del derecho de resistencia de Locke. Si bien Locke defiende el derecho del pueblo «a apelar a los cielos» y revelarse en caso de que el poder legislativo o el ejecutivo quieran esclavizarlo o destruirlo, cabe destacar -como indica Matteucci (op. cit., 135 y 140)- que en Locke, por un lado, la disolución del gobierno por parte del pueblo no implica la disolución de la sociedad política y, por otro, «la acción del pueblo sirve para restablecer el viejo orden legal violado y no para instituir un nuevo orden». Véase también J. Tully (op. cit., 39 y s.).

50 J. Locke, Segundo Tratado, 144.

$51 \mathrm{Ibid}, 160$.

52 Ibid,146. Cabe mencionar aquí la ausencia de referencias al «poder judicial» como «poder independiente» en la teoría constitucional de Locke.

53 J. Locke, Segundo Tratado, 205. Todo lo cual implica además que «independiente- 
monarca ostente los poderes ejecutivo, federativo y de prerrogativa, al tiempo que participa del legislativo. En el Segundo Tratado tal propuesta cobra realidad en diversos pasajes. Así ocurre por ejemplo cuando se refiere a aquellos casos en que los que el ejecutivo reside en manos de una sola persona y ésta forma parte del poder legislativo. ${ }^{54} \mathrm{En}$ estos casos estamos ante una forma de gobierno en la que tal persona (el monarca) también ostentará -como ya hemos dichoel poder ejecutivo, el poder federativo y el de prerrogativa. Pero, de ser así, la «monarquía moderada» de Locke es obviamente menos «equilibrada» de lo que en realidad parece ser, pues presupone una evidente concentración de poderes en manos del monarca. Es más, sugiere -confirmando tal perspectiva- que en estos casos no solo «no hay poder legislativo superior a esta persona» sino que, además, «ninguna ley podrá hacerse sin su consentimiento». ${ }^{55}$ En esa misma línea se pronuncia en otro momento cuando insiste en que el poder ejecutivo «no está exento de subordinación, a menos que dicho poder le haya sido entregado a uno que, al participar del poder legislativo, no tiene por encima a ningún legislador superior al que subordinarse y al que rendir cuentas». ${ }^{56} \mathrm{~A}$ tal hombre -que, como se acaba de sugerir, tendrá en última instancia el poder de vetar las decisiones del legislativo- se le deberá prestar «juramentos de obediencia y fidelidad», pero siempre «de acuerdo con la ley», esto es, siempre que esa persona no viole la ley y actúa conforme al bien común. Así mismo, en el capítulo final del Segundo Tratado Locke se refiere-si bien a título de ejemplo y en un contexto en que aborda la cuestión de la disolución del gobierno- a una forma de gobierno que sin duda expresa con claridad sus aspiraciones a este respecto y, en última instancia, su idea de la «constitución más sabia». Señala en primera instancia que «cuando alguien a quien el pueblo no ha designado para ello asume la función de hacer leyes, las estará haciendo sin autoridad; y, por consiguiente, el pueblo no está obligado a obedecerlas» ${ }^{57}$ Ahora bien, sugiere seguidamente que es difícil determinar quiénes están haciendo mal uso del poder legislativo «sin saber primero bajo qué forma de gobierno sucede» esto. $Y$ es aquí donde Locke ${ }^{58}$ sostiene que acaso lo mejor sea una forma de gobierno en la que el poder legislativo «reside en una concurrencia de tres personas: 1) Una persona individual con carácter hereditario, que tiene permanentemente el supremo poder ejecutivo y, con él, el de convocar y disolver periódicamente

mente de lo que él ordene o haga,..., no será susceptible de que se emplee la fuerza contra él, ni de que sea sometido a censura o condena judicial»»». Con ello Locke defiende el principio de $n$ irresponsabilidad del monarca.

54 J. Locke, Segundo Tratado, 151.

55 Ibid, 151.

56 Ibid, 152 (cursiva nuestra).

57 Ibid, 212.

$58 \mathrm{Ibid}, 213$. 
a las otras dos personas; 2) Una asamblea de la nobleza hereditaria; y 3) Una asamblea de representantes elegidos pro tempore por el pueblo». Con ello Locke no sólo introduce en el poder legislativo a una asamblea hereditaria de carácter aristocrático (la Cámara de los Lores) que limita aún más la soberanía de los representantes elegidos por el pueblo. También termina por perfilar una concepción del «gobierno mixto» que persigue un cierto equilibrio entre diversas clases sociales (monarquía, nobleza y burguesía), pero de la que, no obstante, están excluidas todas aquellas personas a las que, como ya hemos indicado, no se les reconocen derechos políticos. Por otra parte, Locke concibe tal forma de gobierno como una «monarquía moderada» ${ }^{59} \mathrm{o}$, más concretamente, al modo de la «monarquía mixta» ${ }^{60}$ inglesa, a la que considera tan opuesta al despotismo como a la «democracia perfecta». Por eso cree que tal tipo de monarquía es la mejor y más adecuada expresión del «gobierno mixto» en tanto que permite, por una parte, la presencia y equilibrio entre las clases mencionadas, por otro evitar el absolutismo monárquico y, por último, sortear «los peligros de la democracia», esto es, tanto la anarquía y conflictividad social cuanto las pretensiones del pueblo de determinar la forma política del Estado y/o ocuparse de los asuntos de gobierno. ${ }^{61}$

No obstante, y a fin de caracterizar adecuadamente el modelo político lockeano, acaso convenga determinar con mayor precisión el tipo de «equilibrio» social y político que subyace al mismo. Y a tal respecto cabe recordar en este momento la célebre afirmación de Tocqueville según la cual «el gobierno que se llama mixto me ha parecido siempre una quimera. No hay, por decirlo así, gobierno mixto (en el sentido que se da a esta palabra), porque en cada sociedad se acaba por descubrir un principio de acción que domina sobre los demás». ${ }^{62} \mathrm{Y}$, para el caso de Locke y de la forma de gobierno por la que apostó, puede considerarse igualmente acertada la sugerencia de Tocqueville respeto a la Inglaterra de mediados del XIX, esto es, su concepción de la misma como «un Estado esencialmente aristocrático, aunque se encontraran en su seno grandes elementos democráticos; porque las leyes y las costumbres estaban allí establecidas así, de manera que la aristocracia debía siempre, a la larga, predominar y dirigir a su voluntad los negocios públicos»». ${ }^{63}$

59 R. I. Aaron, John Locke. Oxford: Clarendon Press, 1973, 280

60 J.H. Franklin, op. cit., 1978.

61 Locke participa así de aquella «fascinación frente al gobierno mixto» que -al decir de N. Matteucci (op. cit., 104)- dominó la elaboración teórica durante la guerra civil inglesa y los años posteriores.

62 A. de Tocqueville, La democracia en América. México: FCE, 1963, 258.

63 J. Plamenatz sostiene por su parte (op. cit., 1992, 331) que la forma de gobierno de la época «era una mezcla monarquía y aristocracia, y no hay razón para suponer que Locke encontró defectos en ella». 


\section{Conclusiones}

Si tras lo ya señalado volvemos ahora la vista hacia aquella interpretación de Locke como un continuador del radicalismo leveller y como un partidario inequívoco de la igualdad política y la democracia podrán vislumbrarse los problemas que a este respecto ya anunciábamos en los inicios de este trabajo. Y si de algún modo hubiera que resumir tales problemas cabría indicar -con N. Matteucci- que con Locke «del cielo del iusnaturalismo hemos descendido así a la tierra inglesa». ${ }^{64}$ Por lo demás, a las indicaciones hasta ahora realizadas habría que añadir que a estas mismas conclusiones apuntan la misma trayectoria personal de Locke, sus relaciones con Shaftesbury, ${ }^{65}$ su apoyo a la monarquía de Guillermo de Orange ${ }^{66}$ y, en última instancia, el Segundo Tratado. Es cierto que éste puede ser caracterizado como una obra que, «surgida con un motivo ocasional y para un fin contingente», ${ }^{67}$ alcanzó una doble meta: «por un lado, ofrecer un fundamento teórico más firme al constitucionalismo inglés; por otro, convertir la Constitución inglesa en un modelo de organización del poder civil para toda Europa». ${ }^{68}$ Pero, habida cuenta de lo ya señalado y a tenor de aquella interpretación de Locke como un continuador del radicalismo leveller, no es menos cierto que su apuesta por la «monarquía moderada» representa un claro retroceso democrático respecto al modelo constitucional propuesto por aquellos. Y lo representa, en primer lugar, porque Locke -a diferencia del republicanismo de los levellers- es partidario de una monarquía mixta que, por definición, debilita o limita el elemento democrático del poder legislativo; en segundo lugar, y de nuevo a diferencia de los levellers, porque Locke mantiene y preserva como institución fundamental de su propuesta de régimen político a la aristocrática y hereditaria Cámara de los Lores que aquellos querían disolver; en tercer lugar, porque Locke concentra en el monarca los poderes ejecutivo, federativo y de prerrogativa, al tiempo que lo concibe como parte del poder

64 N. Matteucci, op. cit., p 137 y s.

65 Además de lo ya indicado acerca de los vínculos entre Locke y Shaftesbury, conviene recordar ahora que éste último fue un celoso defensor de la monarquía inglesa como modelo de «constitución mixta» bajo la forma de una monarquía limitada por el poder de los Lores y el de los Comunes. Es más, defendió insistentemente el derecho de prerrogativa del rey y los privilegios de los Lores. Véanse a este respecto M. Cranston (op.cit., 105 y ss.) y David McNally (op.cit., 17-40).

66 Recuérdese en este sentido que Locke dedica el Segundo Tratado -como indica en el Prefacio a la primera edición de 1690 de los Dos Tratados- a «establecer el trono de nuestro Gran Restaurador, Nuestro actual Rey Guillermo, para validar su título, el cual siendo el más legítimo de todos, él posee con mayor plenitud y claridad que ningún otro Príncipe de la Cristiandad».

67 N. Matteucci, op. cit., 128.

68 Ibid., 128. Véase también J. Tully, op. cit., 12 y ss. 
legislativo; y, finalmente, porque el tipo de sufragio contemplado por Locke parece incluso más regresivo que el de los levellers. ${ }^{69}$ Así pues, la propuesta política de Locke lejos de desarrollar formas más avanzadas de gobierno representativo se opone a ellas y persigue el retorno a la monarquía moderada.

Por otra parte, y nuevamente frente a otras lecturas de la misma, es cierto que «la obra de los Dos Tratados no es una racionalización de una Revolución [la «Gloriosa»] necesitada de defensa, sino la llamada a una revolución que aún no se había realizado». ${ }^{70}$ Pero es igualmente cierto que, en realidad, esa era la llamada a una «revolución» promovida por una oligarquía propietarista que si, por una parte, se enfrenta a las aspiraciones absolutistas, por otra, se aleja notablemente de los objetivos del «republicanismo popular» $\rangle^{71}$ de los levellers. De hecho, es en parte contra a este último y sus innovaciones sociales e institucionales frente a los que finalmente se organiza y triunfa la Revolución Gloriosa que Locke defendió. Y a este último respecto convendría tener presente que el mismo concepto de «revolución» con que por entonces se designa a tal triunfo se utiliza por primera vez -como ha señalado Hannah Arendt ${ }^{72}$ - en 1660 tras el derrumbamiento del Rump Parlament y la restauración de la monarquía. Pero, a nuestros efectos, es aún de mayor relevancia recordar-con Arendt- que por entonces tal término se utilizaba con «un contenido metafórico que estaba más cerca del significado original del término, ya que servía para designar [no tanto la idea del fin definitivo de un orden antiguo y el comienzo de otro nuevo cuanto] un movimiento de retroceso a un punto preestablecido». De hecho, en cuanto a la Revolución Gloriosa se refiere cabe señalar -insiste Arendt- que ésta «no fue concebida de ninguna manera como una revolución, sino como una restauración del poder monárquico a su gloria y virtud primitivas»». ${ }^{73}$ Por lo demás buena muestra de estas inclinaciones lockeanas es tanto su creencia de que la mejor constitución es aquella que cuenta con una larga y dilatada

69 Cf. C.B. Macpherson (op. cit., 99 y ss.).

70 P. Laslett, «La Revolución Inglesa y los Dos tratados sobre el gobierno de Locke», en J. Locke, Segundo Tratado, XXXIII. Laslett discute aquí con aquellos que -como es el caso de A. MacIntye (Historia de la Ética. Barcelona: Paidós, 1982, 155) o R. I. Aaron (op.cit., 270)sostuvieron que los Dos tratados se publicaron con el propósito de justificar la revolución de 1688

71 S.D. Glover, «The Putney Debates: Popular versus Élitist Republicanism», Past \& Present, 164, 1999, 47-80.

72 H. Arendt, op. cit., 44. Fue entonces -indica Arendt- cuando «encontró su puesto definitivo en el lenguaje político e histórico».

73 Ibid. En opinión de Arendt (op. cit., 56) el concepto de «revolución» solo adquiere las actuales connotaciones de fin de un orden antiguo y comienzo de uno nuevo a partir de la Revolución francesa. 
historia cuanto su equívoca e interesada afirmación -adjudicando al pueblo lo que no parecen ser más que sus opciones e intereses- de que el pueblo siempre es reacio a abandonar sus viejas formas de gobierno o constituciones. Lo que Locke persigue no es pues una revolución constitucional sino, más bien y como se señalaba al inicio de estas páginas, el retorno a la «antigua constitución». Y nada más elocuente a este respecto que su conclusión de que es precisamente esto lo que muestran «las muchas revoluciones que hemos presenciado en este país», esto es, que el mismo pueblo «ha seguido sujetándonos, o, tras algunos infructuosos intervalos, ha vuelto a sujetarnos, a nuestro viejo orden legislativo de rey, lores y comunes». ${ }^{74} \mathrm{Y}$ si esto es así, la reinterpretación de Locke como un demócrata genera no pocas dudas y merece más de un matiz pues los elementos democráticos de la concreta forma de «monarquía moderada» por la que apuesta quedan ampliamente limitados tanto por su estructura, su articulación interna y su modo de funcionamiento cuanto por su concepción de quienes son realmente los ciudadanos o sujetos con plenos derechos políticos.

Roberto Rodríguez Guerra es profesor Titular de Filosofía Moral de la Universidad de La Laguna.

\section{Lineas de investigación:}

$\mathrm{Su}$ labor investigadora ha girado en torno al análisis histórico-crítico del modelo ideo-político liberal, a los desafíos éticos y políticos planteados por el pluralismo y, en la actualidad, sobre la teoría e historia de la democracia.

\section{Publicaciones recientes:}

2013: «El triunfo y las crisis de la democracia liberal», Política y Sociedad, 50, 2, pp. $657-679$.

2013: «De nuevo sobre los problemas de la democracia liberal en el siglo XX», en Ernesto Garzón Valdés, Javier Muguerza, Tony R. Murphy, (comps.), Democracia y participación. Gran Canaria: Fundación Maphre Guanarteme, pp. 85 - 140.

Dirección postal: Universidad de La Laguna. Facultad de Filosofía. Campus de Guajara s/n. C.P. 38200.

Correo electrónico: rrguerra@ull.edu.es

74 J. Locke, Segundo Tratado, 223. 